patients who did not undergo angiography with troponin levels above the ULN (figure 1D). On cox regression analysis, following adjustment for demographic and clinical factors, including troponin level, angiography was associated with a $39 \%$ reduction in mortality during follow-up (hazard ratio 0.61, 95\% CI 0.42-0.89, $\mathrm{p}=0.01$ ).

Conclusion Above the ULN, higher troponin levels were associated with higher mortality. Within the normal range, this was not the case. While angiography was not common in patients presenting with $\mathrm{AF}$, the likelihood of angiography increased at higher troponin levels. In cases where angiography was not performed, there was a direct relationship between troponin levels above the ULN and mortality; this was not evident where angiography was performed. Following adjustment for troponin level, angiography was associated with a significant reduction in mortality. The risk of mortality in patients presenting with AF may be mediated by CAD. Clinical trials are warranted to clarify the investigation and treatment of $\mathrm{CAD}$ in patients presenting with $\mathrm{AF}$ with elevated troponin levels.

Conflict of Interest No conflicts of interest

\section{UTILISATION OF AN EXISTING EXERCISE-BASED CARDIAC REHABILITATION PROGRAMME FOR PATIENTS WITH ATRIAL FIBRILLATION IN THE NATIONAL HEALTH SERVICE: PUTTING CURRENT SERVICES TO GOOD USE}

${ }^{1}$ Mark Mills*, ${ }^{2}$ Elizabeth Johnson, ${ }^{3}$ Hamza Zafar, ${ }^{4}$ Andrew Horwood, ${ }^{5}$ Nicola Lax, ${ }^{6}$ Sarah Charlesworth, ${ }^{7}$ Anna Gregory, ${ }^{8}$ Justin Lee, ${ }^{9}$ Jonathan Sahu, ${ }^{10}$ Graeme Kirkwood,

${ }^{11}$ Nicholas Kelland, ${ }^{12}$ Andreas Kyriacou. ${ }^{1}$ Sheffield Teaching Hospitals NHS Foundation Trust; ${ }^{2}$ Sheffield Teaching Hospital NHS Foundation Trust; ${ }^{3}$ Sheffield Teaching Hospital NHS Foundation Trust; ${ }^{4}$ Sheffield Teaching Hospital NHS Foundation Trust; ${ }^{5}$ Sheffield Teaching Hospital NHS Foundation Trust; ${ }^{6}$ Sheffield Teaching Hospital NHS Foundation Trust; ${ }^{7}$ Sheffield Teaching Hospital NHS Foundation Trust; ${ }^{8}$ Sheffield Teaching Hospital NHS Foundation Trust; ${ }^{9}$ Sheffield Teaching Hospital NHS Foundation Trust; ${ }^{10}$ Sheffield Teaching Hospital NHS Foundation Trust; ${ }^{11}$ Sheffield Teaching Hospital NHS Foundation Trust;

${ }^{12}$ Sheffield Teaching Hospital NHS Foundation Trust

\subsection{6/heartjnl-2019-BCS.30}

Introduction Exercise-based cardiac rehabilitation is an established intervention in the management of several cardiovascular conditions, including ischaemic heart disease and heart failure. There is increasing recognition for the role of exercise-based cardiac rehabilitation in the management of patients

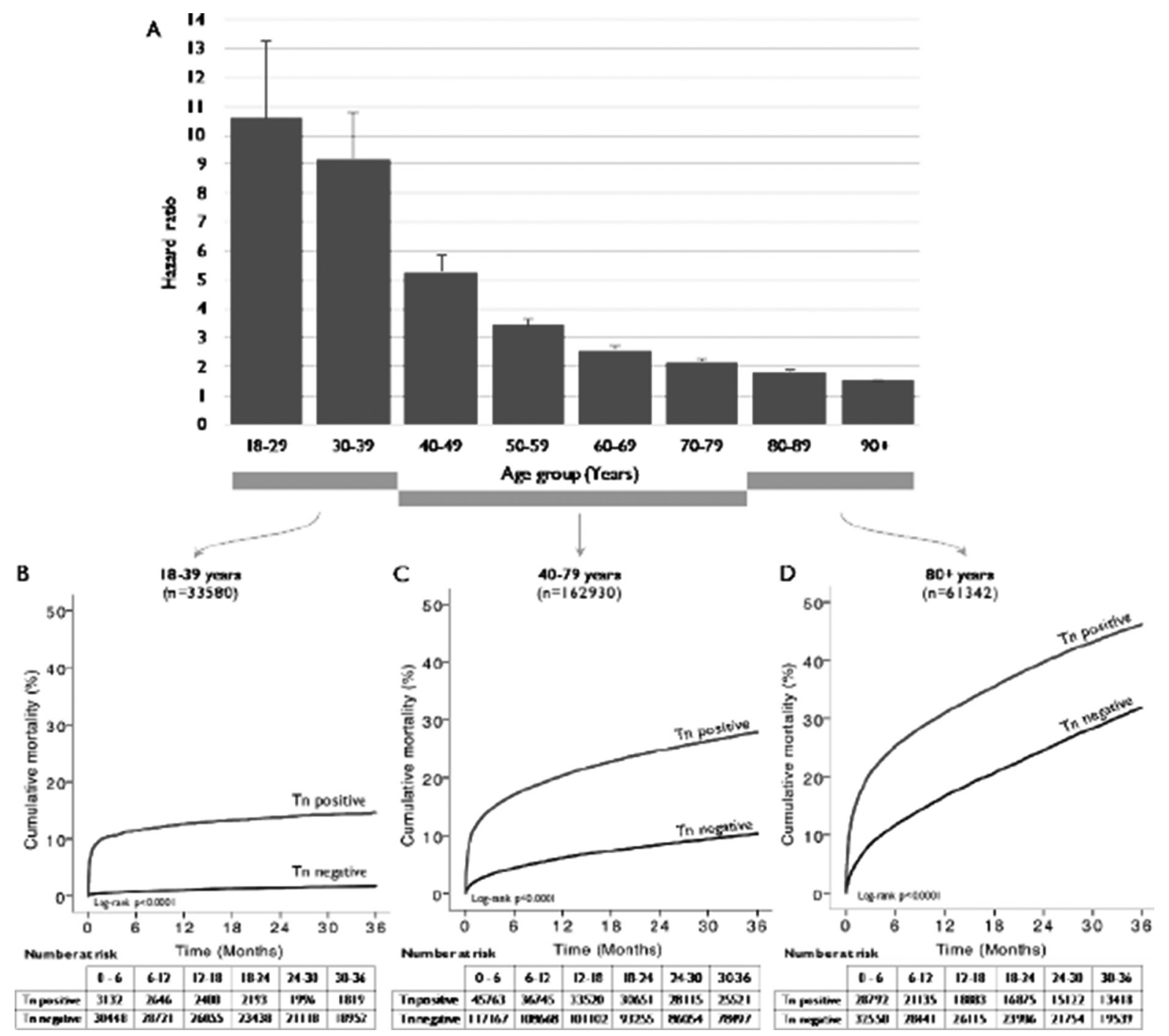

Abstract 30 Figure 1 A, Hazard ratios for troponin positive versus negative groups across different age bands for all patients; B-D, Kaplan-Meier survival curve by troponin positivity in 18-39, 40-79 and $80+$ year age bands.

Error bars denote upper $95 \%$ confidence interval. Tn, troponin. 


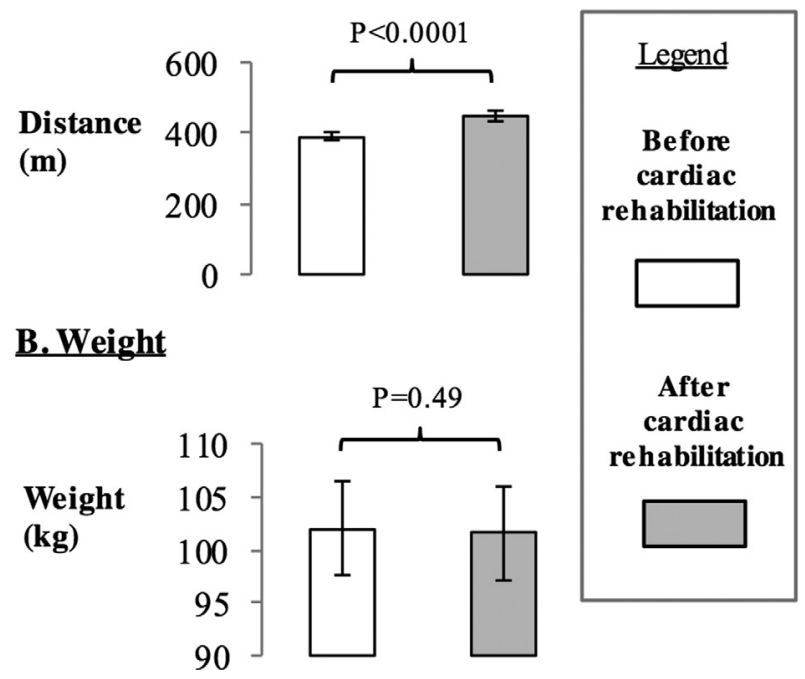

Abstract 31 Figure 1

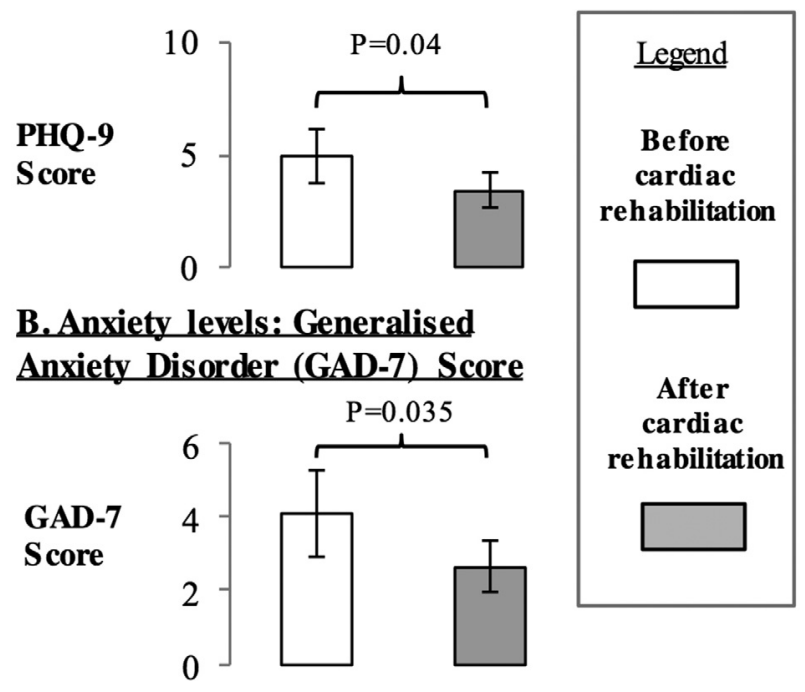

Abstract 31 Figure 2

with atrial fibrillation (AF); however, this has not yet been widely adopted within the National Health Service (NHS). We assessed the feasibility of utilising an established NHS cardiac rehabilitation programme in the management of $\mathrm{AF}$, and examined the effects of this intervention on exercise capacity, weight, and psychological health.

Methods Patients with AF were invited to participate in an established 6-week cardiac rehabilitation programme, composed of physical activity and education sessions, organised through Sheffield Teaching Hospitals NHS Foundation Trust, between April 2016 to July 2018. At the start of the programme, patients were weighed and measured, performed the 6-minute walk test (6MWT), completed the Generalised Anxiety Disorder Questionnaire (GAD-7; scoring 0-21, higher scores indicating higher anxiety levels), and the Patient Health Questionnaire (PHQ-9; scoring 0-27, higher scores indicating higher depression levels). Measurements were repeated on

Abstract 31 Table 1
\begin{tabular}{|l|l|}
\hline $\begin{array}{r}\text { Atrial fibrillation type } \\
-\quad \text { Paroxysmal } \\
-\quad \text { Persistent } \\
-\quad \text { Permanent }\end{array}$ & $\begin{array}{l}15(45.5 \%) \\
16(48.5 \%) \\
2(6 \%)\end{array}$ \\
\hline Hypertension (\%) & $20(60 \%)$ \\
\hline Hypercholesterolemia (\%) & $14(42 \%)$ \\
\hline Heart failure & $5(15 \%)$ \\
\hline Ischaemic heart disease (\%) & $5(15 \%)$ \\
\hline Diabetes (\%) & $5(15 \%)$ \\
\hline Obstructive sleep apnoea & $4(12 \%)$ \\
\hline
\end{tabular}

completion. Analyses were performed using IBM SPSS (24). Continuous variables expressed as mean \pm SEM, and normality of distribution assessed using the Shapiro-Wilk test. Paired continuous data were compared with paired $t$ test or Wilcoxon signed rank test, and unpaired continuous data with independent sample $t$ test or Mann-Whitney $U$ tests, as appropriate. Tests were 2-tailed. $\mathrm{P}<0.05$ was considered statistically significant.

Results Seventy-seven patients were invited to join the programme. Of these, 22 patients $(28.5 \%)$ declined participation prior to initial assessment, whilst $22(28.5 \%)$ accepted and attended the initial assessment, but subsequently failed to attend the programme. In total, 33 patients $(43 \%)$ completed the entire programme $(63.9 \pm 1.7$ years, $58 \%$ female, BMI $33.9 \pm 1.3 \mathrm{~kg} / \mathrm{m}^{2}$, mean left atrial size $\left.5.1 \pm 0.2 \mathrm{~cm}\right)$. Pertinent demographics are summarised in table 1 . On completion, enrolled patients covered longer distances during the 6MWT (389.5 vs. 447.9 metres, $\mathrm{p}<0.0001$; a $15 \%$ improvement), had lower GAD-7 scores (4.12 vs $2.65, \mathrm{p}=0.035)$, and lower PHQ-9 scores (5.0 vs. 3.42, p=0.04). Patient weight was unchanged on completing cardiac rehabilitation $(102.1 \mathrm{~kg}$ vs. $101.6 \mathrm{~kg}, \mathrm{p}=0.49$ ) (Image $1 \& 2$ ). Compared to patients that completed the entire programme, those who attended the initial assessment but failed to complete the programme $(n=22)$ had significantly higher weight and BMI (respectively, 115.6 $\mathrm{kg}$ vs. $102.1 \mathrm{~kg}, \mathrm{p}=0.047 ; 37.9 \pm 2.0$ vs. $33.9 \pm 1.5 \mathrm{~kg} / \mathrm{m}^{2}$, $\mathrm{p}=0.047)$, covered a shorter distance during the 6MWT $(318.8 \mathrm{~m}$ vs. $389.5 \mathrm{~m}, \mathrm{p}<0.01)$, had higher PHQ-9 scores $(9.87$ vs. $5.0, \mathrm{p}=0.037)$, and higher GAD-7 scores (7.53 vs. 4.12, $\mathrm{p}=0.047$ ).

Conclusion Enrolling patients with AF into an established NHS cardiac rehabilitation programme is feasible, with nearly half of those invited completing the programme. This resulted in improved 6-minute walk test, and reduced anxiety and depression levels, in the short term. Severe obesity, high anxiety and depression levels, and lower initial exercise capacity 
may be barriers to enrolling patients with AF into exercisebased cardiac rehabilitation; a holistic approach that targets these factors may result in improved clinical outcomes.

Conflict of Interest None to declare.

\section{ABSTRACT WITHDRAWN}

\section{COST-EFFECTIVENESS OF VENTRICULAR TACHYCARDIA CATHETER ABLATION: LIMITATIONS IN THE CURRENT TRIAL EVIDENCE BASE}

\author{
${ }^{1}$ Yang Chen*, ${ }^{2}$ Manuel Gomes, ${ }^{3}$ Jason Garcia, ${ }^{4}$ Pier Lambiase. 'Barts Health NHS Trust;
}

${ }^{2}$ University College London; ${ }^{3} \mathrm{NHS} ;{ }^{4} \mathrm{UCL}$

\subsection{6/heartjnl-2019-BCS.31}

Introduction Comparative effectiveness research has emerged as a main area of focus in order to highlight and develop more cost-effective evidence based treatments. Within cardiovascular medicine, randomised clinical trials (RCTs) have suffered from criticisms including a lack of generalisability as well as a lack of analysis of the cost-effectiveness of the different interventions being studied. Such analyses are used by organisations including the National institute for Health and Care excellence (NICE) to inform system-level decisions regarding which treatments the NHS should fund. In cardiology, treatments often involve expensive technologies, with the potential for a growing chasm to exist between what is the latest and greatest innovation and what can be afforded on the frontline.

Aim To evaluate the cost-effectiveness of ventricular tachycardia (VT) catheter ablation versus anti-arrhythmic drug (AAD) therapy in ischemic heart disease.

Methods A decision-analytic Markov model was used to calculate the costs and health outcomes of catheter ablation or AAD treatment of VT for a hypothetical cohort of patients with ischaemic cardiomyopathy and an implantable cardioverter defibrillator (ICD). Model inputs where informed using RCT-level evidence [table 1] wherever possible and health states were selected according to evidence of pragmatically measurable differences between each state [figure 1]. Health states that did not have any patientreported HRQL data supporting the calculation of a QALY value were excluded. Costs were calculated from a UK perspective.

Results Catheter ablation versus AAD therapy had an incremental cost-effectiveness ratio (ICER) of $£ 144,150$ $(€ 161,448)$ per quality adjusted life year (QALY) gained, over a five-year time horizon [table 2]. The ICER for a tenyear time horizon was $£ 75,074(€ 84,083)$ and $£ 69,986$ $(€ 78,384)$ over the cohort's lifetime. Using probabilistic sensitivity analyses to account for model parameter uncertainty,
A
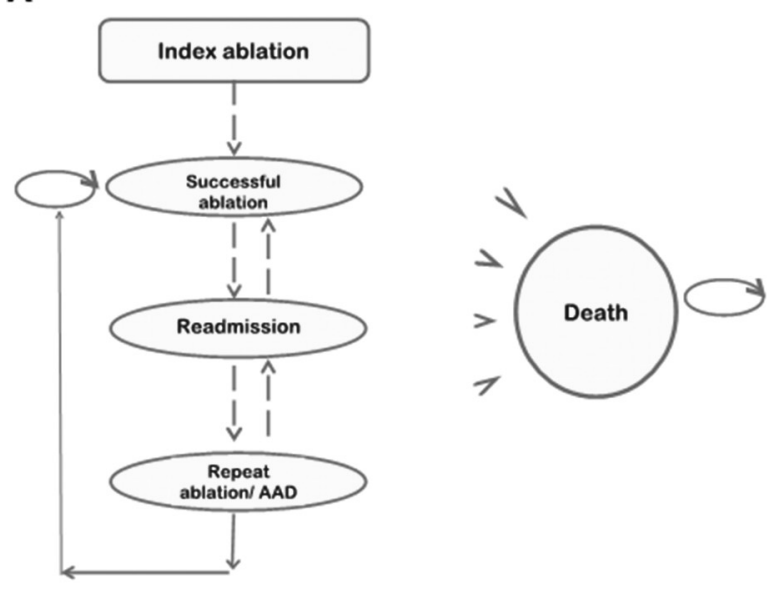

B
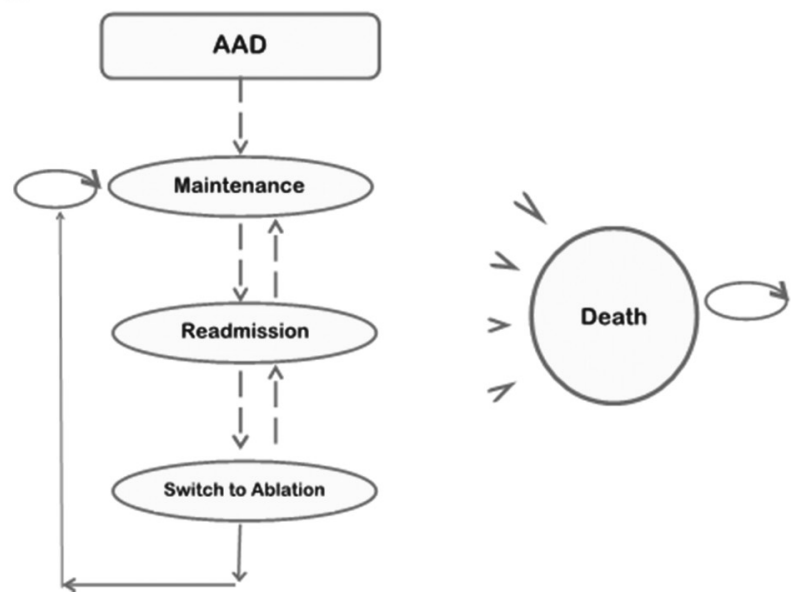

Abstract 33 Figure 1 Schematic of model structure used in simulation $A$ ) represents the model for the ablation arm B) represents the model for the AAD arm

the likelihood of catheter ablation being cost-effective was only 11\%, assuming a willingness to pay threshold of $£ 30,000$ used by the NICE [figure 2]. One-way sensitivity analyses suggested that cost-effectiveness inferences were robust to a wide range of departures from base-case assumptions, including changes in baseline mortality, procedural mortality and readmission rate.

Conclusion Catheter ablation of VT is unlikely to be costeffective compared with AAD therapy alone in patients with ischaemic cardiomyopathy implanted with an ICD based on pooled trial evidence. However, better designed studies incorporating detailed and more frequent quality of life assessment are needed to advise health policy in this field and to provide more informed cost-effectiveness analyses.

Conflict of Interest None relevant 